\title{
Music Playlist Recommendation Based on User Heartbeat and Music Preference
}

\author{
Hao Liu, Jun Hu , Matthias Rauterberg \\ Industrial Design Department \\ Technical University of Eindhoven \\ Eindhoven, Netherlands \\ \{ hao.liu, j.hu, g.w.m.rauterberg \}@tue.nl
}

\begin{abstract}
In this paper, we present a new user heartbeat and preference aware music recommendation system. The system can not only recommend a music playlist based on the user's music preference but also the music playlist is generated based on the user's heartbeat. If the user's heartbeat is higher than the normal heartbeat which is 60-100 beats per minutes (age 18 and over) or $70-100$ beats per minutes (age 6-18), the system generates a user preferred music playlist using Markov decision process to transfer the user's heartbeat back to the normal range with the minimum time cost; if the user's heartbeat is normal, the system generates a user preferred music playlist to keep the user's heartbeat within the normal range; If the user's heartbeat is lower than the normal heartbeat, the system generates a user preferred music playlist using Markov decision process to uplift the user's heartbeat back to the normal range with the minimum time cost.
\end{abstract}

Keywords-music playlist recommendation; heartbeat; music preference; markov decision process

\section{INTRODUCTION}

Everyone has a normal heartbeat rhythm. The rhythm varies from person to person. For a healthy person, his/her heart at rest beats about 60 to 100 times per minutes (age 18 and over) or 70 to 100 times per minutes (age 6-18). If his/her heart beats fast or slow and the rhythm is disrupted, it may cause serious health problems such as lightheadedness, dizziness and fainting, etc. In the literature, music is used to keep a person's heartbeat at normal range. Knight and Rickard revealed that relaxing music attenuated blood pressure and heart rate after a stressful task; moreover, the level of subjective anxiety was reduced after the presentation of relaxing music [1]. Stratton and Zalanowski conducted experiments and found that preference, familiarity or past experiences with the music have an overriding effect on positive behavior change than other types of music [2].

However, with technology improvements in networks, storage, portability of devices, etc., personal accessible music collections have grown exponentially in recent years. This situation makes it difficult for users to browse and discover their favorite music from the vast amount of music collection by themselves. As an alternative, music recommend systems are being pursued to recommend users preferred music without "unnecessary” user explicit inputs. Much work has been done on developing music recommendation systems over the last decade. The interest in this area still remains high because it constitutes a typical problem rich recommendation application, and because of its promising commercial prospects that help the listener to find preferred music or promote new music to the listener.

In this paper, we present a new linear biofeedback web-based music system. It integrates the concepts of adaptive systems, user music preference, and methods of using the music to adjust the user's heart rate into a linear biofeedback control system. The system can not only recommend a music playlist based on the user's music preference like a traditional music recommendation system, but also the recommended music playlist is generated based on the user's heartbeat to keep his/her heart beating within the normal range. If the user's heartbeat is higher/lower than the normal heart rate, the system generates a user preferred music playlist using Markov decision process to lower/uplift the user's heartbeat back to the normal range with the minimum time cost; if the user's heartbeat is normal, the system generates a user preferred music playlist to keep the user's heartbeat within the normal range.

The rest of this paper is organized as follows. Related works are discussed in Section 2. Our new user heartbeat and preference aware music recommendation system is presented in Section 3. In section 4, we introduce the architecture of our new music recommendation system. Then, the user experiment method and result are presented in section 5 to validate our music recommendation system. Conclusions are presented in Section 6.

\section{RELATED WORKS}

In this section, firstly, the current music recommendation systems are investigated. After that, the current methods of using music to manipulate the user's heartbeat in the music therapy field are investigated.

\section{A. Current Music Recommendation Systems}

Today, with the explosive growth in the amount of available digital music, music recommendation systems have become an important research area. There has been much work done both in industry and academia on developing new approaches to music recommendation systems over the last decade [3] [4] [5] [6].

Currently, there are two types of architecture for music recommendation systems. Figure 1 presents type one architecture. In this architecture, if the user wants to get desired music services for recreation, he/she needs to interact with the music system explicitly to browse and select the desired music services from the provided options. The 
Liu H.,Hu J., Rauterberg M. (2009). Music Playlist Recommendation Based on User Heartbeat and Music Preference. In: Proceeding of 2nd IEEE 2009 International Conference on Computer Technology and Development (pp.545-549), 2009. IEEE Press ISBN: 978-0-7695-3892-1.

system can recommend music based on the user's explicit interaction with the system. Examples of architecture one include [3] [4]. Figure 2 introduces architecture two. Compared to architecture one, the user's implicit input is used to facilitate personalized music recommendation and decrease the unnecessary dialogues between the user and the system. Examples of architecture two include [5] [6].

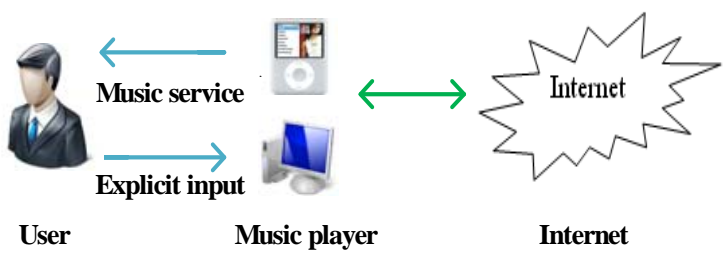

Figure 1. Architecture of music recommendation systems.

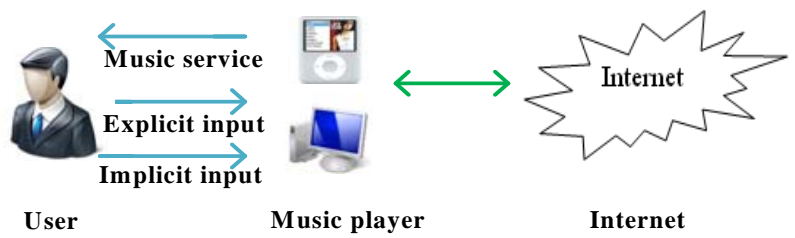

Figure 2. Architecture of music recommendation systems.

\section{B. Current Music Heartbeat Adjust Methods}

To my best knowledge, there are few literatures about relations between the heart beat and music tempo. Peter Sleight found that listening to music with a slow or meditative tempo has a relaxing effect on people, slowing breathing and heart rate. Listening to faster music with a more upbeat tempo has the opposite effect - speeding up respiration and heart rate [7]. However, there is a long literature involving the use of music for reducing the user's stress. Researches have show that heart rates can be used as an indicator of stress. For example, Nancy found that during the exam and when their graded exams were returned to them, most students' heart rates increase substantially [8]. Taelman reported that a person's heart rate is significantly faster when he is in mental stress [9].

Miluk-Kolasa et al. showed that music was one of the relaxing adjuncts in modulating the ascent of autonomic responses to negative stress [10]. Knight and Rickard revealed that relaxing music attenuated blood pressure and heart rate after a stressful task; moreover, the level of subjective anxiety was reduced after the presentation of relaxing music. The tempo of the music being listened to appears to be an important parameter here [1]. Steelman looked at a number of studies of music's effect on relaxation where tempo was varied and concluded that tempos of 60 to 80 beats per minute reduce the stress response and induce relaxation, while tempos between 100 and 120 beats per minute stimulate the sympathetic nervous system [11]. White and Shaw reported similar results and argued that tempos slower than the average human's heart rate ( 40 to 60 beats per minute) induce suspense, while tempos of 60 beats per minute with a low pitch are most soothing [12]. Iwanaga found that people prefer music with tempo ranging from 70 to 100 per minute which is similar to that of adults' heart rate within normal daily situations [13].

\section{MusiC Playlist RECOMMENDATION BASED ON USER HeArtbeat and Music PREFERENCE}

In this section, we first introduce the model of a healthy heartbeat, then, the description of the music and user's music preference are presented; after that, a new linear biofeedback based music recommendation system which is used to mediate between the available music, user's music preference, the current heartbeat and the target heartbeat is introduced. Finally we present how to generate a music playlist using Markov decision process to transfer the user from the abnormal heartbeat state to the normal state with the minimum time cost.

\section{A. User Heartbeat Model}

Heart rate is a measure of the number of heart beats per minute. For a child (age 6-18), his/her normal heart rate at rest is 70-100 beats per minute. For an adult (age 18 and over), his/her normal heart rate at rest is $60-100$ beats per minute. If the heartbeat rhythm is disrupted and is beating lower than 60 (age 18 and over) or 70 beats per minute (age 6-15), it is called a bradycardia. A slow heart beat may cause dizzy spells, blackouts, breathlessness or tiredness. If the heart beats higher than 100 beats per minutes, it is called a tachycardia. When the heart beats rapidly, the heart pumps less efficiently and provides less blood flow to the rest of the body, including the heart itself. The increased heart rate also leads to increased work and oxygen demand for the heart (myocardium), which can cause a heart attack (myocardial infarction) if it persists. This occurs because the decreased flow of necessary oxygen to the heart causes myocardial cells to begin to die off.

From the above description, then, for each age group, we can model three heartbeat states: tachycardia (100-220), normal (60-100 or 70-100) and bradycardia (60-0 or 70-0).
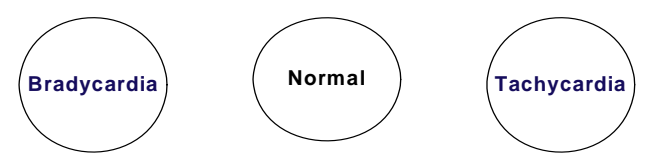

Figure 3. Healthy heartbeat model 
Liu H.,Hu J., Rauterberg M. (2009). Music Playlist Recommendation Based on User Heartbeat and Music Preference. In: Proceeding of 2nd IEEE 2009 International Conference on Computer Technology and Development (pp.545-549), 2009. IEEE Press ISBN: 978-0-7695-3892-1.

\section{B. Music Metadata}

Music Metadata are data that are used to describe the characteristics of the music. ID3 [14] is a metadata container most often used in conjunction with the MP3 audio file format. It allows information such as the title, artist, album, track number, tempo or other information about the file to be stored in the file itself. In this paper, our music system implementation is based on ID3 music Metadata.

\section{User Music Preference Model}

User music preference includes not only the user's long term evolving commitment to certain categories of music service but also user's spontaneous music service requirement which depends on context of use [15]. However, Rentfrow found that when people discuss their music preferences they tend to do so first at the level of genres and to lesser extent subgenres and only later step up to broader terms (e.g., loud) or down to specific artists (e.g., Van Halen) or songs (e.g., "Running with the Devil” ) [16]. In this paper, for simplicity reasons, the genre which is the user's long term music preference is the optimal level at which to start our investigations of music preferences.

\section{A New Linear Bio-feedback Based Music Recommendation System}

The objective of the music recommendation system is depicted in figure 4, If the user's heartbeat is in a bradycardia state, the system should generate a user preferred music playlist to uplift the user's heartbeat back to the normal state with the minimum time cost; If the user's heartbeat is in a tachycardia state, the system should generate a user preferred music playlist to calm the user's heartbeat back to the normal range with the minimum time cost; if the user's heartbeat is normal, the system generates a user preferred music playlist to keep the user's heartbeat within the normal range.

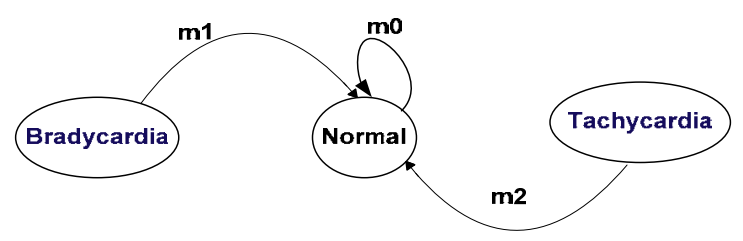

Figure 4. Heartbeat transition with music playlist

Figure 5 presents our new music system framework. In the figure, the framework starts by setting the user's target normal heartbeat state. Then, the system begins observing the passenger's current heartbeat state (modeled on the passenger's heart rate feedback signals) that it wishes to control. This step of perception creates an internal representation of the passenger's heartbeat situation. After that, depending on the difference between the target and the current real heartbeat state, the inference component in the framework must determine (1) whether the user is in the target state or not; and (2) if the passenger is not in the target state then an optimized music playlist is recommended based on user music preference and available music etc. information to transfer the passenger from the current state to the target state to promote his/her heart health. The user himself/herself is an adaptive system; his/her perception creates an internal representation of the music. This perception affects the user's internal heart state. During this process, the passenger's heartbeat states may also influenced by the set of variables which in the control system called disturbances. The change in the passenger's heartbeat states is again perceived by the system, and this again triggers the adaptation process we have described, thus closing the control loop.

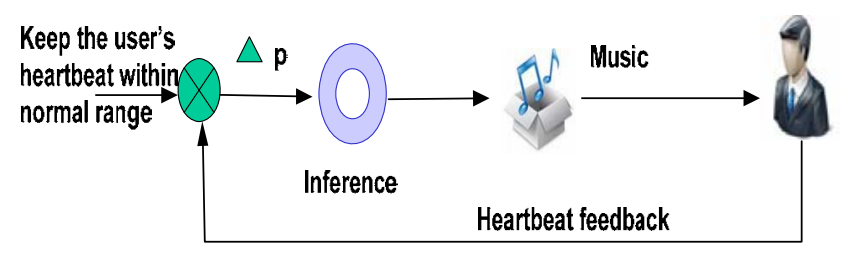

Figure 5. A new music recommendation system framework

\section{E. Music Playlist Generation Using Markov Decision Process}

From sub section 3.4, we can see that inference is the central part of our music recommendation system. Its functionality is that if the user's heart beats out of the normal range, the system generates a user preferred music playlist to transfer the user's heartbeat back to the normal with the minimum time cost using Markov decision process.

A few definitions need to be defined to use Markov decision process to optimize music playlist recommendation to transfer the passenger from the current state to the target state with minimum time cost.

Definition 1: A passenger's heartbeat state can be formally expressed as $S=\left\{s_{1}, S_{2} \ldots s_{m}, \ldots s_{n}\right\}$ where $1 \leq m \leq n$ and $S_{m}$ is one of his/her possible heartbeat states. In this paper's context, $n$ equals 3 .

Definition 2: Inference action space $A$ is formally expressed as $A=\left\{a_{1}, a_{2} \ldots a_{m}, \ldots a_{n}\right\}$ where $1 \leq m \leq n$ and $a_{m}$ is one category of its recommended music (actions) which could enable the passenger's heartbeat state transition.

Definition 3: A transition probability function $P_{a}\left(s_{i}, s_{j}\right)$ is defined as the probability that action $a$ in state $s_{i}$ at time $t$ will lead to state $s_{j}$ at time $t+1$. It can be formally represented as $P_{a}\left(s_{i}, s_{j}\right)=\operatorname{Pr}\left(s_{t+1=} s_{j} \mid s_{t}=s, a_{t}=a\right)$. 
Definition 4: $R_{a}\left(s_{i}, s_{j}\right)$ is defined as the time reward $T_{i j}$ received after transition to state $s_{j}$ from state $s_{i}$ with transition probability $P_{a}\left(s_{i}, s_{j}\right) . T_{i j}$ equals $-f_{m}$ where $f_{m}$ is the time cost of transferring the passenger state from $S_{i}$ to $S_{j}$.

Definition 5: A transition probability matrix $P$ is defined as

$$
\begin{aligned}
& P=\left(\begin{array}{ccc}
P_{11} & \ldots & P_{1 n} \\
\vdots & \ddots & \vdots \\
P_{n 1} & \cdots & P_{n n}
\end{array}\right) \text { where } \\
& P_{i j}=P_{a}\left(s_{i}, s_{j}\right)=\operatorname{Pr}\left(s_{t+1=} s_{j} \mid s_{t}=s, a_{t}=a\right) .
\end{aligned}
$$

Based on the above definitions, the optimized music playlist recommendation to transferring the user heartbeat state from current heartbeat state $S_{i}$ to the target heartbeat state $S_{m}$ with the minimum time cost can be solved with the Bellman equation:

$v\left(s_{i}\right)=\max _{a \in A}\left\{R_{a}\left(s_{i}, s_{i}\right)+r \sum_{s_{j} \in S} P_{a}\left(s_{i}, s_{j}\right) v\left(s_{j}\right)\right\} \quad$ where $r$ is the discount rate and satisfies $0<r \leq 1$. If we want the passenger to transfer from current heartbeat state $s_{i}$ to the target heartbeat state $S_{m}$ with minimum intermediate states, $r$ could be tuned smaller. Otherwise $r$ could be tuned towards 1. By this Bellman equation, the inference can compute an optimized action (music playlist) list to transfer the passenger from the current heartbeat state to the target $\mathrm{s}$ heartbeat state with minimum time cost. For example, if the user's heart rate is in a tachycardia state, then the inference generates a Music playlist which is composed by user preferred music with tempos ranking from 60 to 80 [17].

\section{IMPLEMENTATION}

\section{A. The Architecture of the Music System}

Figure 6 shows the main components that make up our biofeedback based music system. The whole architecture is divided into five abstraction levels from the functionality point of view. The lowest level is the resource level which contains the music, heart rate bio sensor and user music preference. The second layer is the resource manager layer which includes the music service manager, bio signal manager and user music preference manager. The music manager is responsible for music registration, unregistration, etc. functions. The bio signal manager collects and models signals from bio sensors and stores them in the database. The user music preference manager collects and updates the user's music preference information. The third layer is the database layer which constitutes by a database. It acts not only as a data repository, but also enables the layers and the components in the layers loosely coupled. This increases the flexibility of the whole architecture. For example, replacing or updating components in the resource manager layer does not affect the architecture performance unless data structures they store in the database changed. The fourth layer is the adaptive control unit layer which includes inference component. It is used to mediate between the music, heart rate state and available music to recommend personalized music to transfer the passenger from the current state to the target state. The fifth layer is the interface layer. The passenger interacts with the music system interface to get music services.

The music system is implemented with web-based browse/server architecture. The user can install the music system in a computer (server). He/she can then browse the music system via the internet. The advantage of this structure is that the user can manage his/her music collection centrally and can access it in difference situations and locations over the internet. If the user wants to use the music system at some locations (office, home, etc.), he/she just need to install fix heart rate sensors at those locations or bring a mobile heart rate sensor with him/her.

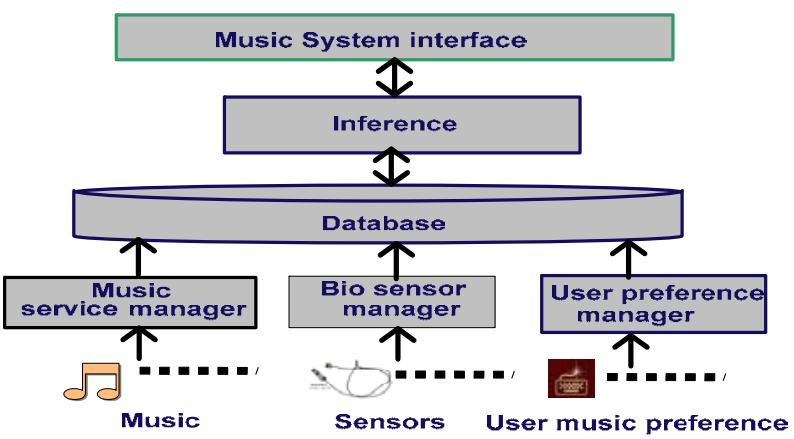

Figure 6. The architecture of the music system

\section{EXPERIMENT}

An experiment to transfer the user from a bradycardia state to the normal state has been done in the main building of the technical university of Eindhoven, for group one, six people's heartbeats are monitored in a seat which embedded a non intrusive heart rate sensor at 9:30-9:40, 12:20-12:30, and 17:00-17:40. The subjects' ages range from 25 to 35 which include three female and three male. The experiment result shows that all the subjects' heart rates are lower at 12:00-12:30 and 17:00-17:40 than at 9:30-9:40. Four of them are lower than the normal heart rate at 17:00-17:40 or/and 12:00-12:30 depending on the intensity of their workload. For group two, it is composed of the same group of people in group one, firstly, their heart beats are measured between 
Liu H.,Hu J., Rauterberg M. (2009). Music Playlist Recommendation Based on User Heartbeat and Music Preference. In: Proceeding of 2nd IEEE 2009 International Conference on Computer Technology and Development (pp.545-549), 2009. IEEE Press ISBN: 978-0-7695-3892-1.

17:00 and 17:10, the results show that their heartbeat are lower than their normal heartbeat at 9:30-9:40, three of them are in bradycardia states, then an user preferred music playlist using Markov decision process is generated and we ask the subject to listen to it for thirty minutes, the experiment result show that all the subjects' heartbeat can transfer back to the normal heartbeat after listening to the recommended music playlists.

We are now doing the experiment of transferring the user from a tachycardia state to the normal state. The tachycardia state is simulated by asking the user to use the home trainer to uplift his/her heart rate.

\section{CONCLUSION}

In the music therapy field, music plays an important role in improving user's heart health. At the same time, the current music recommendation systems have made significant progress in recommending context-aware and personalized music from a large collection. However, few works have been done to design music recommendation systems to recommend user preferred music to keep his/her heartbeat within normal range. In this paper, we present a new bio feedback based music recommendation system. Compared to the current music recommendation system, it can regulate the user's heartbeat within normal range with user preferred genre of music. We have already implemented our bio feedback based music system and done the experiment of uplifting the user from a bradycardia state to the normal state with his/her preferred music. The result partly validates the feasibility of our system design. Now, we are doing the experiment of transferring the user from a tachycardia state to the normal state.

\section{REFERENCES}

[1] WEJ. Knight and NS. Rickard, "Relaxing Music Prevents Stressinduced Increases in Subjective Anxiety, Systolic Blood Pressure, and Heart Rate in Healthy, Males and Females”, Journal of Music Therapy, 38(4), pp. 254-72,2001.

[2] VN. Stratton and AH. Zalanowski, "The Relationship between Music, Degree of Liking, and Self-Reported Relaxation”, Journal of Music Therapy, 21(4): 184-92, 1984.
[3] Pandora, "Personalized music service”, retrieved May 1, 2009 from Pandora's Web site: http://www.pandora.com.

[4] NH. Liu, SW. Lai, CY. Chen and SJ. Hsieh, "Adaptive Music Recommendation Based on User Behavior in Time Slot", IJCSNS International Journal of Computer Science and Network Security, VOL.9 No.2, pp 219-227, 2009.

[5] Jarno Seppänen, Jyri Huopaniemi, "Interactive and context-aware mobile music experiences", Proc. of the 11th Int. Conference on Digital Audio Effects (DAFX-08), Espoo, Finland, September 1-4, 2008

[6] J. Wang, MJT Reinders, J. Pouwelse, RL. Lagendijk, "Wi-Fi walkman: a wireless handhold that shares and recommends music on peer-to-peer networks" IS\&T/SPIE Symposium on Electronic Imaging 2005.

[7] L. Bernardi, C. Porta, P. Sleight, "Cardiovascular, cerebrovascular, and respiratory changes induced by different types of music in musicians and non-musicians: the importance of silence”. Heart (British Cardiac Society). 92(4):445-52, 2006.

[8] L. Nancy Elwess and F. Daniel Vogt, "Heart Rate and Stress in a College Setting”. Bioscience, Volume 31(4):pp 20-23, December 2005

[9] J. Taelman, S. Vandeput, A. Spaepen and S. Van Huffel, "Influence of Mental Stress on Heart Rate and Heart Rate Variability". J. Vander Sloten, P. Verdonck, M. Nyssen, J. Haueisen (Eds.): ECIFMBE 2008, IFMBE Proceedings 22, pp. 1366-1369, 2008.

[10] B. Miluk-Kolasa, M. Matejek and R. Stupnicki, “ The Effects of Music Listening on Changes in Selected Physiological Parameters in Adult Pre-surgical Patients”. Journal of Music Therapy, 33, pp 208218, 1996.

[11] VM. Steelman, "Relaxing to the beat: music therapy in perioperative nursing”. Today's OR Nurse, Vol. 13, pp.18-22,1991.

[12] J. White and C. Shaw, "Music therapy: a means of reducing anxiety in the myocardial infarction patient". Wisconsin Medical Journal, July, 90(7), pp. 434-437, 1991.

[13] M. Iwanaga, "Relationship between heart rate and preference for tempo of music”. Percept Mot Skills, Oct, 81(2):435-40, 1995.

[14] Wikipedia, “ID3”. Retrieved March 1, 2009 from Wiki’s Web site: http://en.wikipedia.org/wiki/ID3.

[15] H. Liu, B. Salem, M. Rauterberg, "Adaptive User Preference Modeling and Its Application to In-flight Entertainment" . In: Proceedings of 3rd International Conference on DigitalInteractive Media in Entertainment and Arts, ACM ISBN: 978-1-60558-248-1, pp. 289-294, 2008.

[16] P. J. Rentfrow, S. D. Gosling: “ The do re mi’s of everyday life: The structure and personality correlates of music preferences”. Journal of Personality and Social Psychology, 84, 1236-1256, 2003.

[17] H. Liu, J. Hu, M. Rauterberg, "AIRSF: A New Entertainment Adaptive Framework for Stress Free Air Travels” . In: Masa Inakage \& Adrian David Cheok (eds.): Proceedings of the International Conference on Advances in Computer Entertainment Technology, pp. 183-186,2008. 